\title{
Longitudinal, Regional and Deformation-Specific Corpus Callosum Shape Analysis for Multiple Sclerosis
}

\author{
Omer Ishaq, Ghassan Hamarneh, Roger Tam and Anthony Traboulsee
}

\begin{abstract}
The corpus callosum (CC) is an anatomical structure which connects the two brain hemispheres. Neurological diseases can cause atrophy of the $\mathrm{CC}$ resulting in a change in its size and shape. The measurement and analysis of this change is one of the goals of clinical research. We perform statistical analysis of the shape of the $\mathrm{CC}$ extracted from MR brain scans of a group of multiple sclerosis patients undergoing a longitudinal (serial) study. In contrast to the classical boundary-based, global shape variability measures, e.g. principal component analysis (PCA) of CC boundary vertices, we perform a deformation-specific PCA for analyzing the global and regional shape of the $\mathrm{CC}$. This deformationspecific PCA is based on a medial-based shape representation. The adopted shape representation describes shape variability in terms of intuitive deformations (e.g. bending, stretching and thickness). We present qualitative and quantitative results for 412 MR images of the $C C$. We show that our method is successful in identifying and quantifying the effect of each type of deformation on the shape variability of the $\mathrm{CC}$. In addition to analyzing the spatial shape variability in the $\mathrm{CC}$, we explore shape changes as the disease progresses. Our method allows the exploration of the shape variability quantitatively (e.g. the amount of variance explained by a particular principal mode of shape variation) as well as in a qualitative visual manner (e.g. by visualizing, say, the $2^{\text {nd }}$ principal mode of shape variation due to bending at the $4^{t h}$ sub-region of the $\mathbf{C C}$ ) which is useful for developing an intuitive understanding of the effects of MS on the CC shape.
\end{abstract}

\section{INTRODUCTION}

The corpus callosum (CC) is an anatomical structure of non-uniform cross-section, which is identified in a migsagittal MR image (figure 1). The CC connects the two brain hemispheres by acting as a bridge for the nerve fibers. These nerve fibres transmit neural impulses between the hemispheres. Multiple Sclerosis is an inflammatory and degenerative disease of the central nervous system and is associated with brain atrophy. This atrophy can result in a change in the size and shape of the CC [1]. Considering the importance of the $\mathrm{CC}$ for interhemispheric communication, one of the goals of clinical research has been to find the size and shape changes taking place in the $\mathrm{CC}$ for longitudinal studies of patients with neurological diseases. Consequently, a large volume of research has focused on the analysis of the change in CC shape [4-12]. However, most of these studies suffer from the following problems. First, most of these studies are based on either a global shape analysis of the $\mathrm{CC}$, which fails to capture the regional shape changes, or

This research was funded by grants from MS/MRI, MITACS and NSERC. O. Ishaq and G. Hamarneh are with the Medical Image Analysis Lab, School of Computing Science, Simon Fraser University, Burnaby, Canada.

R. Tam and A. Traboulsee are with the Division of Neurology, Department of Medicine, University of British Columbia, Vancouver, Canada. use a regional representation that is not clinically meaningful. Second, the few studies which have analyzed the CC shape on the basis of a clinically meaningful CC subdivision scheme are neither longitudinal nor based on in-vivo scans of the patients [3].

In this paper we make following contributions: (i) we perform longitudinal analysis of CC shape changes for MS patients; (ii) we analyze a relatively large dataset of MR scans $(\mathrm{N}=412)$. This data is also quite suitable for a longitudinal study in terms of the time frame, as it spans 300 days, which is a clinically significant amount of time for observing changes due to MS; (iii) we examine CC shape variability at multiple scales (global and regional) and locations and our subdivision of the CC is based on a clinically meaningful subdivision scheme [3]; (iv) we examine the shape changes by decomposing the shape variability into intuitive shape deformations, such as stretch, bend and thickness of the CC, using a medial axis based representation [2] and by performing a deformation-specific principal component analysis (PCA) for each deformation. Unlike previous work [2], there is no overlap in the shape variations due to these different deformations and these variations can be linearly added to obtain the total variation in the dataset; (v) we quantify and visualize the shape variations, both spatially and temporally, due to the principal modes of variation for each of the deformations at the different subdivisions of the CC.

\section{METHODS}

\section{A. Shape Representation Using Medial Profiles}

The CCs in 2D mid-sagittal MR scans of the human brain were first expert segmented. Following skeletonization and pruning of the segmented data, a medial based shape representation, 'medial profiles', is calculated for each CC [2]. We use medial profiles representation because it follows the geometry of the $\mathrm{CC}$ and allows for the description of the $\mathrm{CC}$ shape in terms of four intuitive and independent shape measures (profiles). These are the stretch $S$, bend $B$, inner thickness $I$ and outer thickness $O$ profiles. Further, this representation allows for the decomposition of the $\mathrm{CC}$ shape variability into different intuitive deformation types (stretch, bend, and bulge) thus enabling us to perform statistical analysis of the shape deformation at different locations and for different regions of the $\mathrm{CC}$ for each deformation type. These profiles also allow for the reconstruction of the $\mathrm{CC}$ shape. By controlling the number of medial nodes we can capture the CC shape with different levels of details. The characteristics captured by these four profiles (i.e., stretch, 
bend, inner thickness and outer thickness) can also be described as deformations in shape. For details of the medial profiles shape representation the reader is referred to [2].

\section{B. Deformation Specific Statistical Analysis}

Our goal is to calculate the statistical influence of each deformation on the variation in CC shape. In this manner we will be able to capture the deformation with the largest contribution to the variation in global or regional CC shape for longitudinal studies. To this end, we perform a deformationspecific PCA of the CC shape which captures the shape variation due to the specific deformation. To perform this deformation-specific PCA we use the four profiles (stretch, bend, inner thickness and outer thickness) that were extracted for each of the CCs. We calculate four mean profiles $\bar{S}, \bar{B}$, $\bar{I}$ and $\bar{O}$. These mean profiles can be used to reconstruct a mean CC shape, which is the mean shape of the whole data set of the segmented CCs. In order to perform a PCA specific to one of the deformations, say a stretch specific PCA, we set the other three profiles of each CC to their mean values, i.e. $B=\bar{B}, I=\bar{I}$ and $O=\bar{O}$, and the stretch profile $S$ for each CC is left unchanged. These profiles are used to reconstruct the boundary of the CCs. All the CCs reconstructed in this manner have the same average bend, inner and outer thickness. The variation in the shape of these CCs is only due to the variation in their stretch. We perform a PCA on the boundary points of these reconstructed CCs. The eigenvalues from this PCA correspond to the magnitude of the principal modes of shape variation specific to only one deformation; stretch, in our example. The sum of these eigenvalues $\Gamma^{S}=\sum_{k=1}^{t} \lambda_{k}^{S}$, where $\lambda_{k}^{S}$ is the $k^{t h}$ eigenvalue for the stretch deformation, provides the magnitude of total variation due to the stretch deformation. Similarly we obtain $\Gamma^{B}, \Gamma^{I}$ and $\Gamma^{O}$ for the other three deformations. We then find the percentage contribution of each deformation to the total $\mathrm{CC}$ shape variation present in our dataset. The contribution of the stretch deformation to the total variation in $\mathrm{CC}$ shape can be written as $\Gamma^{S} /\left(\Gamma^{S}+\Gamma^{B}+\Gamma^{I}+\Gamma^{O}\right)$. Similarly we calculate the percentage contributions of the other three deformations. We perform this analysis and find the variation in both the global (section II-C) and regional CC shape (sections II-D and II-E).

\section{Longitudinal Analysis of the Global CC Shape}

We perform a longitudinal analysis of the change in the global CC shape. The longitudinal MR scans for each patient are sorted into three temporal bins. The first bin contains the baseline scans and the scans performed within the first 100 days of the baseline scan. The second bin contains the scans performed between 100 and 200 days from the baseline scan. The third bin contains the scans performed between 200 and 300 days of the baseline scan. On average each patient underwent seven scans in the 300 days after his/her first scan. For each bin, we perform the deformation-specific PCA and calculate the percentage contribution of each deformation to the variation in the global CC shape, as explained in II-B.

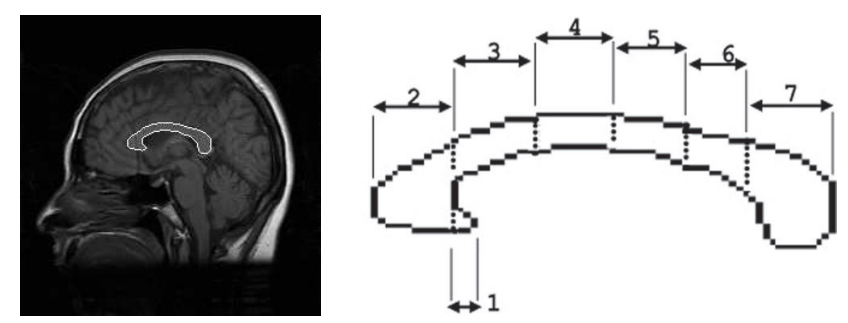

Fig. 1. CC in the human brain outlined in white on a mid-sagittal MR image (left). The Witelson subdivision of the CC (right).

\section{Shape Analysis of the Witelson-Based CC subdivisions}

We perform a non-longitudinal shape analysis of the regional change in the $\mathrm{CC}$ shape. Each $\mathrm{CC}$ is divided into seven subdivisions. This division is based on the established Witelson framework which divides the CC into seven subdivisions according to specific geometric constructs [3]. The subdivisions of a sample CC are shown in figure 1. Next, we obtain four 'sub-medial profiles' for each CC subdivision. We perform a deformation-specific PCA for each CC subdivision and calculate the percentage contributions of each deformation to the variation in its shape.

\section{E. Longitudinal Analysis of the Witelson CC subdivisions}

We also perform a longitudinal analysis of the regional change in the CC shape. The CCs are subdivided using the Witelson framework as well as sorted into temporal bins based on the scan date. In this manner, a combination of the techniques in sections II-C and II-D is utilized. We perform the deformation-specific PCA for each CC subdivision in each temporal bin and calculate the percentage contributions to the variation in its shape.

\section{RESULTS}

We present quantitative and qualitative results of our experiments. The experiments were conducted on 2D midsagittal MR brain scans from a longitudinal study involving 412 scans of 51 MS patients over a period of 300 days from the baseline scan. The quantitative results are displayed in figures 2, 3 and 4, where figures 2 and 3 present results for the longitudinal analysis of global and regional CC shape, respectively, and figure 4 presents results for a non-longitudinal analysis of the regional CC shape. Figure 2 displays the percentage contribution of the first three principal modes due to the four deformation types to the variation in the global CC shape for the longitudinal study. The first three images (from left to right) represent the first three principal modes of variation and the fourth image is the aggregate of the first three modes. Figure 3 shows the percentage contribution of the deformations to the shape variation in the Witelson based $\mathrm{CC}$ subdivisions for a longitudinal study. The first two rows represents the first two modes of variation and the third row is the aggregate of the first two modes. The columns (from left to right) in figure 3 represent the seven Witelson subdivisions. We observe in figures 2 and 3 that the CC stretch increases and the $\mathrm{CC}$ bending decreases with time. 

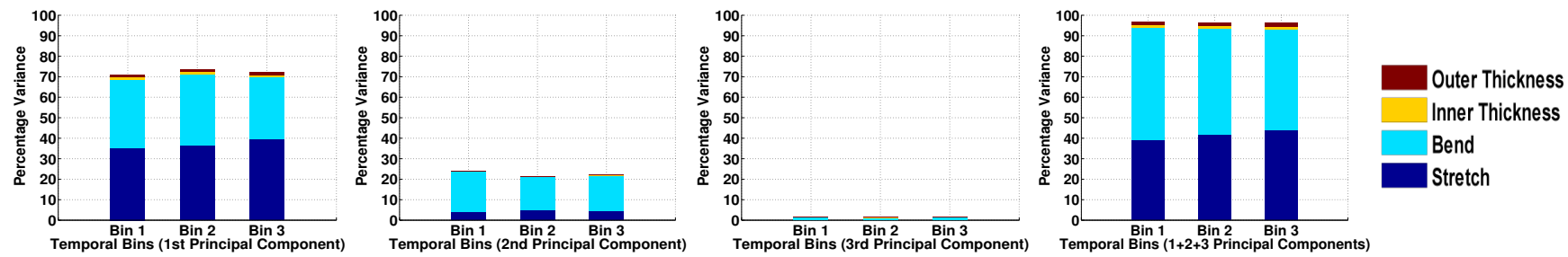

Fig. 2. Percentage contribution of the deformations to the variability in the global CC shape, for a longitudinal study. The first three images (from left to right) represent the results for the first three principal modes of shape variation. The fourth image (far right) is the aggregate of the first three principal modes. The three bars in each image represent the three temporal bins. The first bin contains the baseline scans and the scans performed within the first 100 days of the baseline scan. The second bin contains the scans performed between 100 and 200 days from the baseline scan. The third bin contains the scans performed between 200 and 300 days of the baseline scan. The color coding of each bar displays the individual percentage contribution of each deformation to the shape variation. The legend for the color codes is available at the far right of the figure.
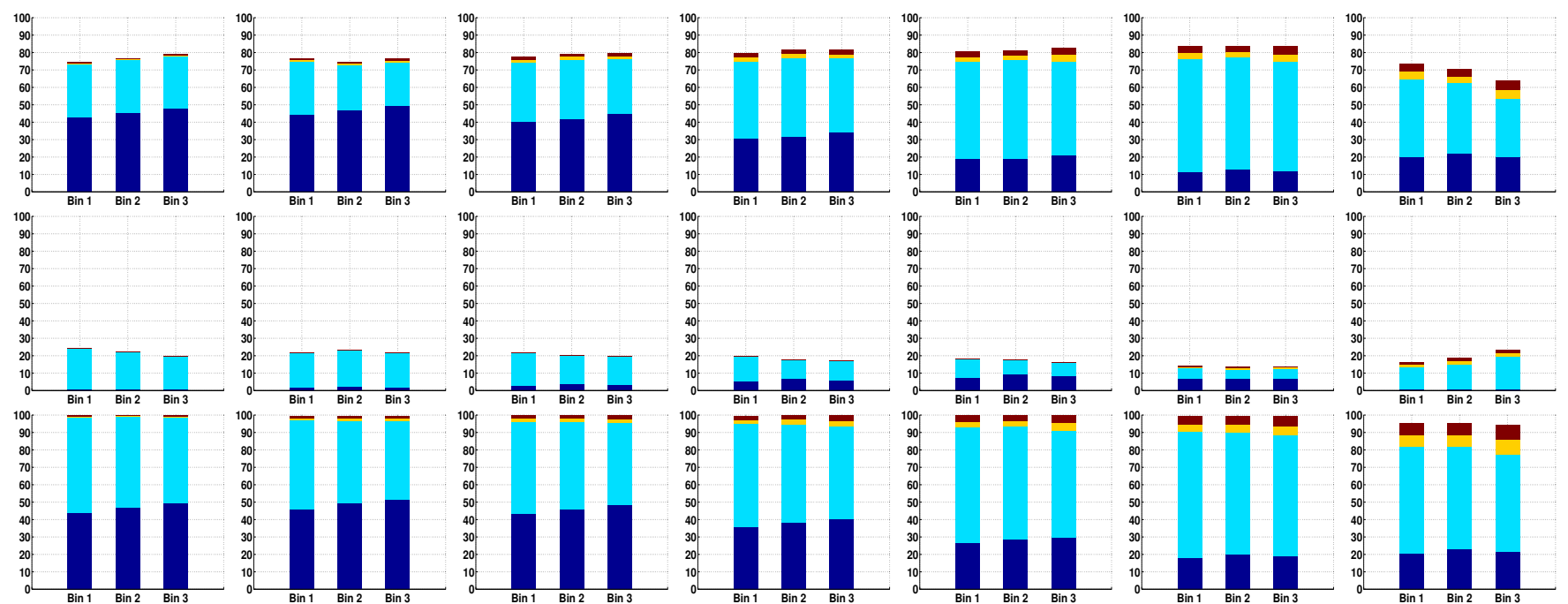

Fig. 3. Percentage contribution of deformations to the shape variability of the Witelson CC subdivisions, for a longitudinal study. The columns represent the seven Witelson subdivisions. In each column the first two rows represent the first two principal modes of shape variation. The third row is the aggregate of the first two principal modes. The bars and the color coding follow the same scheme as in figure 2 .
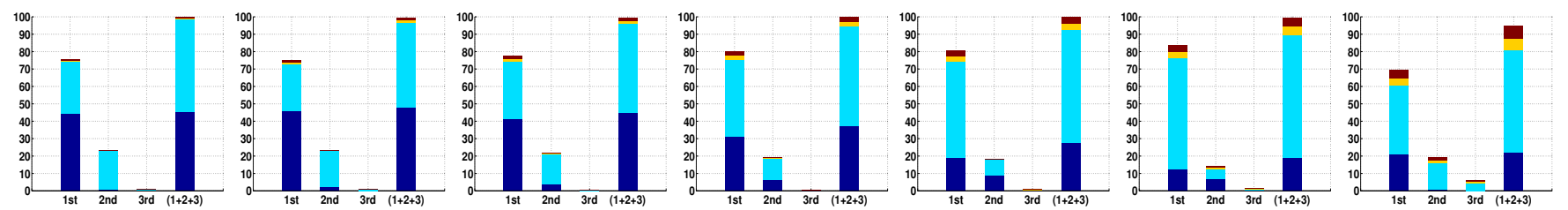

Fig. 4. Percentage contribution of the deformations to the shape variability of the Witelson CC subdivisions, for cross-sectional study of inter-individual variability. The seven images (from left to right) represent the seven Witelson regions. In each image the first bar represents the sum of percentage contributions of the first principal mode of variation for all four deformations. Similarly the second and the third bars represent the sum of the contributions of the second and third principal modes, respectively. The fourth bar represents the aggregate contribution of the first three bars. The color coding scheme is same as in the previous figures.

This suggests that the $\mathrm{CC}$ shape tends to straighten out with the progression of disease and loses some of its curvature. We also observe from row 1 in figure 3 that the effect of the first principal mode of variation due to the deformations tends to increase with time for almost all CC subdivisions, but this effect is particularly more pronounced for the first subdivision. Figure 4 displays the percentage contribution of the deformations to the shape variation in the Witelson based CC subdivisions for a cross-sectional study. The seven images (left to right) represent the seven Witelson subdivi- sions. The results show that the stretch deformation has a more dominant contribution in the first three $\mathrm{CC}$ subdivisions $(\sim 40 \%)$ as compared to the last four subdivisions $(\sim 30 \%)$. It is also of note that the contribution of the inner and outer thickness tend to increase as we move towards the latter half of the CC. The contribution of the bend deformation is maximum for the $6^{\text {th }} \mathrm{CC}$ subdivision. Generally, we observe that the effect of the stretch tends to decrease and the effects of the bend, inner and outer thickness tend to increase in the latter half of the CC. In figure 5 we qualitatively 

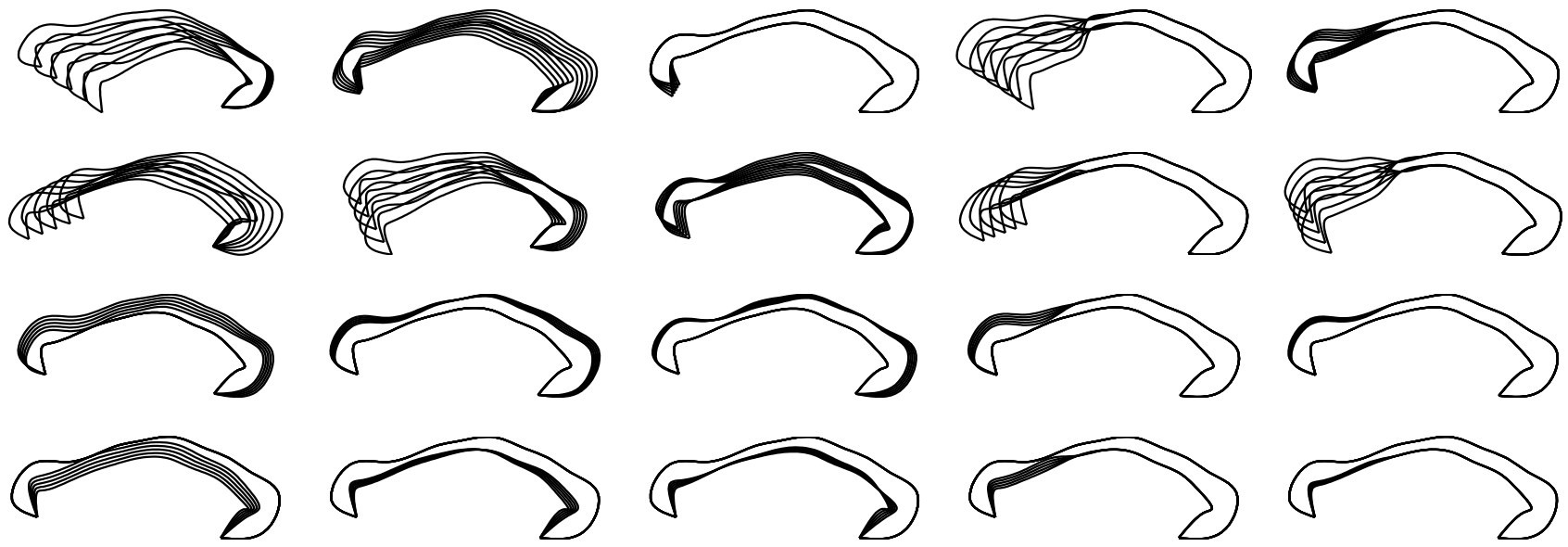

Fig. 5. Reconstruction of the global and regional CC shape variation for the four deformations for cross-sectional analysis of inter-individual variability. The four rows represent the CC shape variation corresponding to the four deformations i.e. stretch, bend, inner thickness and outer thickness. The first column represents the CC shapes reconstructed from varying the first principal mode of shape variation for the global CC shape over standard deviation values ranging from -2.0 to 2.0 in steps of 1.0. The second and the third columns represent the second and third principal modes of shape variation for the global CC shape. The fourth and the fifth column represent the first and second principal modes of shape variation for the regional CC shape.

present the variation in the global and regional CC shape by reconstructing the CCs for different principal modes of shape variation. The four rows represent the four deformations. The first column represents the $\mathrm{CC}$ shapes reconstructed from varying the first principal mode of global CC shape variation over standard deviation values from -2.0 to 2.0 in steps of 1.0. The second and the third columns represent the CC reconstructions for the second and third principal modes of the global shape variation, respectively. The fourth and the fifth column represent the first and second principal modes of shape variation for the regional CC shape. The reconstructed CCs provide a useful visualization of the trends that were observed in the quantitative results of the earlier figures, e.g. it is clear that whereas stretch is dominant primarily for the first mode of variation, the effect of the bending is well distributed for both first and second modes and is in fact the largest contributor of shape variation for the second mode. It is also evident that stretch and bending contribute more to the variation in $\mathrm{CC}$ shape than inner and outer thickness, which is a validation of the earlier plots.

\section{DISCUSSION}

We have performed a medial-based global and regional statistical analysis of the CC shape. We have shown that the adopted shape representation allows for an intuitive description of shape variability. In addition to decomposing shape variability spatially, we have also explored the temporal CC shape changes. We have analyzed the shape variability both qualitatively and quantitatively. Our results have shown that the effect of the four deformations on the shape variation is significantly different for the various CC subdivisions and this effect changes with the progression of the pathology. We have observed that stretch and bending of the CC have a more dominant influence on the variation in $\mathrm{CC}$ shape. Future work includes the correlation of clinical MS measures with CC shape changes.

\section{REFERENCES}

[1] A. Paolillo, C. Pozzilli, C. Gasperini, E. Giugni, C. Mainero, S. Giuliani, V. Tomassini, E. Millefiorini, S. Bastianello, "Brain atrophy in relapsing-remitting multiple sclerosis: relationship with 'black holes', disease duration and clinical disability", Journal of the Neurological Sciences, 174(2):85-91, 2000.

[2] G. Hamarneh, R. Abu-Gharbieh, T. McInerney,"Medial profiles for modeling deformation and statistical analysis of shape and their use in medical image segmentation", International Journal of Shape Modeling, 10(2):187-209, 2004.

[3] S. F.Witelson, "Hand and sex differences in the isthmus and genu of the human corpus callosum”, Brain, 112:799-835, 1989.

[4] J.E. Downhill Jr, M.S. Buchsbaum, T. Wei, J. Spiegel-Cohen, E.A. Hazlett, M.M. Haznedar, J. Silverman, L.J. Siever,"Shape and size of the corpus callosum in schizophrenia and schizotypal personality disorder", Schizophrenia Research, 42(3):193-208, 2000.

[5] F. Robichon, P. Bouchard, J.F. Dmonet, M. Habib, "Developmental dyslexia: re-evaluation of the corpus callosum in male adults", European Neurology, 43(4):233-237, 2000.

[6] P.W. Woodruff, I.C. McManus, A.S. David, "Meta-analysis of corpus callosum size in schizophrenia", Journal of Neurology, Neurosurgery, and Psychiatry, 58(4):457-61, 1995.

[7] A.Y. Hardan, N.J. Minshew, M.S. Keshavan, "Corpus callosum size in autism", Neurology, 55(9):1425, 2000.

[8] S. Malobabic, D. Bogdanovic, G. Teofilovski, "Morphology of the human corpus callosum: the shape of its mediosagittal section", Gegenbaurs morphologisches Jahrbuch, 133(3):403-10, 1987.

[9] L. Hong, J. Blumenthal, L. Clasen, A. Lausier, J. Giedd, "Using multidimensional scaling to assess shape differences of human corpus callosum ”, Proc ICONIP, 219-221, 2002.

[10] G. Hamarneh, J. Chen, N. Lifshitz, M. Henkelman, "Local volume changes of the corpus callosum from 3D MR images of wildtype and knockout mouse brains", Proc ISMRM, 2003.

[11] P. Golland, W.E.L. Grimson and R. Kikinis, "Statistical shape analysis using fixed topology skeletons: corpus callosum study", Proc IPMI, 1999.

[12] C. Davatzikos, M. Vaillant, S. Resnick, J.L. Prince, S. Letovsky, R.N. Bryan, "A computerized approach for morphological analysis of the corpus callosum", Journal of Computer Assisted Tomography, 20:8897, 1996. 